\title{
Transforming mobility systems with sharing and automation
}

\author{
Amanda Stathopoulos ${ }^{1} \cdot$ Ipek N. Sener $^{2}$
}

Published online: 11 September 2017

(C) Springer Science+Business Media, LLC 2017

Rapid changes are underway in urban mobility systems around the world, including the introduction of shared mobility solutions, mobility on-demand and fielding of progressively more automated vehicles. These developments offer prospects of tackling congestion, pollution and mobility accessibility concerns. At the same time, the emergence of new systems poses major behavior and governance challenges. The pace of the introduction and the extent of the effects from these mobility transformations will vary by context and will likely be driven by factors such as urban density, socio-demographic profiles, infrastructure design, technology evolution and changes in mobility attitudes and habits over time.

This special issue gathers papers that focus on recent tools, models and empirical investigations to better inform the modeling and management of future transportation systems, increasingly impacted by on-demand organization, shared vehicles and automated mobility solutions. The special issue gathers papers from the Transportation Research Board (in collaboration with the Standing Committees on Travel Behavior and Values [ADB10] and Demand Forecasting [ADB40]) as part of a long-standing collaboration with Transportation. Our special issue presents five notable papers that were originally presented at the 2017 TRB Annual Meeting.

The papers were selected to represent the state-of-the-art contributing to a growing body of literature to inform travel models and real world applications with a variety of methodological approaches and case studies from three continents. The first three papers center on advancing our understanding of Autonomous Vehicles (AVs), while the

Amanda Stathopoulos

a-stathopoulos@northwestern.edu

Ipek N. Sener

i-sener@tti.tamu.edu

1 Department of Civil and Environmental Engineering, Northwestern University, Evanston, IL, USA

2 Texas A\&M Transportation Institute, Austin, TX, USA 
remaining two cover recent developments in car-sharing, ride-sourcing and multi-modal mobility hub analysis.

The selected studies on automation examine likely evolutions of future societies as the penetration of self-driving vehicles increases. The main challenge lies in combining realistic behavioral factors with the variety of technological and policy options that planners and city managers might pursue. Together, the studies offer new insights on possible effects on travel demand with an emphasis on differences across user segments.

The first paper by Liu and colleagues on "Tracking a System of Shared Autonomous Vehicles across the Austin, Texas Network Using Agent-Based Simulation" presents largescale micro-simulation of transportation patterns in a metropolitan area when introducing a system of shared autonomous vehicles (SAVs). The simulation uses MATSim and draws on land development patterns, demographics, network and trip pattern data from the Capital Area Metropolitan Planning Organization (CAMPO) region of Texas, including the city of Austin. Parameters found in relevant literature along with behavioral assumptions are used to define the allowable waiting time and fare sensitivity related to adopting SAV systems. The study makes trip count predictions where demand is split between SAVs, human-driven cars and public transit, according to underlying utility functions among two groups of travelers (with or without access to a private vehicle). The scenario-testing focuses on the critical issue of SAV fare structure and how that results in changes in spatial trip demands. As expected, there is a general negative correlation between fare and SAV trip rates. More specific findings are observed when differentiating the type of users. In particular, when SAV rates are low, long-distance travelers are particularly likely to select SAVs over other modes. The result is plausible considering that automation decreases the burden of travel, in particular for longer trips. Taken together, the findings suggest that future SAV fare policies could crucially impact SAV distances travelled, competition with transit, empty miles generation, and mobility of travelers that are currently unwilling or unable to drive. The authors conclude by delineating future avenues for research that might reveal novel insights, including allowing for more variation in the willingness to wait or pay for SAV vehicles and studying evolving forms of ownership. The new research avenues would need to be matched with detailed trip rate and destination data to correctly assess the mobility and sustainability impacts of (shared) autonomous vehicles.

The second paper by Truong et al. is titled "Estimating the Trip Generation Impacts of Autonomous Vehicles on Car Travel in Victoria, Australia". The study explores the potential impacts of AVs on overall car travel drawing on empirical data from the Victorian Integrated Survey of Travel and Activity (VISTA) across the Greater Melbourne area. The research seeks to shed light on an often observed challenge related to AVs, namely that providing improved mobility to groups that are hitherto unable to drive might increase overall mileages and hence the negative effects associated with driving. The authors suggest that induced demand, by those who are too young or old to drive, is still a poorly understood issue when discussing the impact of AVs. The paper puts forth a new method to estimate entirely new trips associated with AVs across seven life stages, ranging from infancy to late adulthood. Estimates of new trips are derived from the concept of mobility gaps, that is, differences between needs and actual travel rates of road users at different life stages using assumptions drawn from VISTA data. A daily car trip model is developed where various AV scenarios are tested, varying the mode shifts from public transport and active modes, along with car occupancy rates. The model suggests that AVs will increase car trips, and that the induced trip rates vary quite significantly by age segment. The findings also indicate that increases in AV travel could be magnified by continuing trends of reduced car occupancy rates. Troung and colleagues conclude by 
discussing the most relevant additions to the framework. This includes looking at partial $\mathrm{AV}$ market penetration rates (as opposed to 100\%) and variation in fare structure, and extending the analysis to look at trip mileage, in addition to trip counts.

Taken together, the first two papers in the special issue call for future frameworks that include more behavioral details to further study the joint effect of both shifted and generated travel, as a function of AV penetration in the presence of different user segments and automation scenarios.

The third paper titled as "Literature Review on Surveys Investigating the Acceptance of Autonomous Vehicles" complements the previous papers by surveying the extant literature. Becker and Axhausen's overview of the user motivation and behavior perspective is derived from a literature review of 17 surveys on AVs between 2012 and 2016 included in academic papers and industry reports. In addition to comparing the general methods and results from the surveys, the paper categorizes the surveyed explanatory variables according to their influence in different experiments. In terms of main response variables, the level of acceptance, the modal split, the willingness to pay for automation, and the choice of owning an AV or using a shared (taxi) service are found to be the most common. Instead, more long-term effects, such as relocation choices and induced future travel, is still at an early stage of investigation. Of particular interest is the identification of which explanatory variables are most influential. The geographical spread of studies, along with four cross-national surveys, permits initial observations on the geographical variation. The intention to use the technology varies among different countries, with lower interest in industrialized countries on the whole, and strong alignment among the US studies around $40 \%$ approval rates. Considering the explanatory factors, every survey included sociodemographic variables. The various studies agree that younger respondents and men are more likely to accept AVs. Considering attitudinal factors, studies consider a variety of effects, including personality, locus of control perception and technology awareness. The lack of uniformity makes drawing conclusions challenging. Finally, considering the effect of current mobility behavior, the studies concur that the current vehicle's technology sophistication is a strong predictor of interest in automation. In contrast, current mileage and car-sharing experience are not clear predictors. The paper concludes by identifying several areas that would benefit from further investigation. Concerning the choice between ownership and shared use of AVs, the paper calls for a deeper investigation of the decision unit and how the (family) decision process works. Safety is another factor that has unclear connections to automation interests. Additionally, the passion for driving may induce a lowered interest in automation, except if driving conditions are too poor to justify selfdriving. Therefore, it is important that future surveys increase the level of comparability among effects, to consolidate the findings so far in the literature and to account for the continuous evolution in technology and user motivations.

While autonomous vehicles are not yet available to the public, mobility-on-demand services are rapidly in use around the world, and might keep growing especially with the introduction of autonomous vehicles. Motivated by this growing interest but also the lack of information on their adoption and use in the market, the fourth paper by Dias et al., presents analysis of two disruptive mobility services, entitled "A Behavioral Choice Model of the Use of Car-Sharing and Ride-Sourcing Services". Indeed, there have been some preliminary illustrations of the use of these services, but the studies have mostly relied on aggregate data and methodologies. Diaz and colleagues contribute to the field by developing a disaggregate choice model that jointly models individuals' frequency of ridesourcing and car-sharing use through a bivariate ordered probit form. Using the 2014-2015 Puget Sound Regional Travel Study data, they examine the impacts from various observed 
factors on individuals' decision to use car-sharing and ride-sourcing while also controlling for the unobserved lifestyle preferences that may affect their choices. In general, the results indicate a higher tendency of usage among young, well educated, higher income, and employed individuals as well as the ones residing in higher density neighborhoods. Interaction effects were also found to be quite important, e.g. for the presence of children or the effect of built environment, on the usage level of mobility services. Based on their results, the authors emphasize the need to treat the two novel mobility services as separate modes of transportation in land use and transportation planning as they may have various different implications. For instance, the mobility services may help lower use of private vehicles, but they may also draw away from walking, biking and public transport in high density areas. While providing valuable insights and potential in predicting service usage under different scenarios, this study also suggests improvements in capturing the supplyside of the equation (e.g. cost and travel time). As emphasized by the authors, future research would benefit from studies at the tour or trip level considering both demand and supply factors in an effort to develop robust forecasts of the impacts of such transformative technologies.

Without doubt, multimodality is a key concept to strengthen the positive impacts of transformative mobility options. The fifth and the final paper of this issue on "Impacts of a Multimodal Mobility Service on Travel Behavior and Preferences: User Insights from Munich's first Mobility Station" tackles the mobility services question from this perspective, and presents results from a case-study of a multimodal mobility hub, which are also known as Mobility Stations in Germany. In their study, Miramontes et al. examine the use, perceptions and impacts of the first Mobility Station in Munich. In designing and conducting their study, the authors consider the important role of integrated multimodal systems' ability to compete against the flexibility and convenience of private automobiles. This study can then also be considered as responding to the call of Dias et al. by emphasizing the need to develop and study these mobility options as separate but integrated choices. The authors follow a systematic process by first conducting interviews with stakeholders of the Mobility Station to identify their goals, interests and experiences as the service providers. The insights from these interviews are then used to develop two online surveys. The first survey focuses on the users of the Mobility Station. For their second survey, the authors work with public transport users who have not used the other options of the Mobility Station to understand the causes of non-use and improve the multimodal uptake. Finally, they conduct focus groups to examine the surveys' findings. In their paper, Miramontes and colleagues mainly present the first survey and its results, which highlight various interesting observations about the users, their experiences, awareness and perception, their level of multimodality and mobility patterns. Looking at the user profile, the results show consistent trends with the US study of Dias et al. by pointing to a young and well educated population. The German study also emphasizes the central role of public transport for the majority of users suggesting some degree of multimodality (e.g. use of bike share and car-share) among the users. The results underline several other promising insights, such as the importance of intermodal connections to improve multimodality and the interest among users to have different mobility options for an increase in multimodality. The survey is limited by the reliance on a unique and small sample of users, which calls attention to the need for more user surveys or panel studies focusing on multimodality mobility services. The authors also emphasize the importance of evaluation studies based on both users' and non-users' perspective as well as the implications for upscaling the mobility services to different environments with varying supply and land use characteristics. 
To summarize, smartly integrated, close proximity mobility options can provide multimodal choices without drawing demand from each other, and hence might have more potential to cope with the negative consequences of automobile-based mobility.

Amanda Stathopoulos is an Assistant Professor of Civil and Environmental Engineering at Northwestern University and holds the Junior William A. Patterson Chair in Transportation. Her research focuses on theory and empirical models of mobility behavior and citizen acceptance of transformation in mobility systems. Recent projects have analyzed the human dimensions in defining the impact of innovative mobility systems such as crowd-sourced and shared transportation.

Ipek N. Sener is an Assistant Research Scientist at the Texas A\&M Transportation Institute. Her main research focus is on transportation modeling and travel behavior analysis. She has a particular interest in transportation-based health research, promoting sustainable and equitable environments, and studying the health outcomes of transportation systems, the built and travel environment, and individuals' activity-travel behavior. She is also dedicated to exploring the effect of information and communication tools on travel behavior and how to better integrate technology-enabled mobility options. 